\title{
Ion-Beam-Induced Desorption as a Method for Probing the Stability of the Molecule-Substrate Interface in Self-Assembled Monolayers
}

\author{
Sabina Wyczawska, ${ }^{[a]}$ Piotr Cyganik, ${ }^{*[b]}$ Andreas Terfort, ${ }^{[c]}$ and Peter Lievens ${ }^{*[a]}$
}

During recent years self-assembled monolayers (SAMs) have been recognized as model structures for different areas in nanotechnology, ranging from nanolithography and molecular electronics to sensors and biocompatible materials. ${ }^{[1]}$ A growing range of possible new SAM applications demands better understanding of their detailed molecular structure, which is a result of balancing molecule-molecule and molecule-substrate interactions in the monolayer. Despite the numerous structural studies of SAMs available nowadays, the structure and stability of the SAM-substrate interface is still poorly understood. Even for a most simple SAM system of methanethiol on $\mathrm{Au}(111)$, identification of the adsorption geometry (the Au-S interface) remains controversial. ${ }^{[2,3]}$ As a consequence, the experimental and theoretical analysis of the bonding geometry and the stability of the molecule-substrate interface for technologically relevant, and therefore more complicated SAMs, is extremely difficult. Importantly, this missing information remains fundamental for most of SAMs potential applications with molecular electronics in particular. ${ }^{[4]}$

Herein we propose using ion-induced desorption in combination with neutral fragment mass spectrometry as a method to analyze the chemical stability of the molecule-substrate interface in complicated and technologically relevant SAMs. This work builds on previous studies analyzing the behavior of SAMs on a $\mathrm{Au}(111)$ substrate upon bombardment with beams of $\mathrm{Ar}^{+}$ions in the keV range. ${ }^{[5-8]}$ It was shown that the bombardment of aromatic thiol SAMs on a $\mathrm{Au}(111)$ substrate results in the ejection of neutral molecular fragments via two distinct desorption mechanisms. A minor percentage of the SAM fragments leave the surface with high kinetic energy $(\sim \mathrm{eV})$ as a result of the direct momentum transfer from the collision cascade developed in the substrate upon primary ion impact. The overwhelming majority of desorbing particles leaves the surface with low kinetic energies $\left(\sim 10^{-2} \mathrm{eV}\right)$ as a result of "gentle" cleavage of chemical bonds (with no significant momentum transfer) within the organic layer by chemical reactions initiat-

\footnotetext{
[a] Dr. S. Wyczawska, Prof. P. Lievens

Laboratory of Solid State Physics and Magnetism

Katholieke Universiteit Leuven K.U. Leuven

Celestijnenlaan 200D, 3001 Leuven (Belgium)

E-mail:Peter.Lievens@fys.kuleuven.be

[b] Dr. P. Cyganik

Smoluchowski Institute of Physics

Jagiellonian University

Reymonta 4, 30-059 Krakow (Poland)

E-mail:piotr.cyganik@uj.edu.pl

[c] Prof. A. Terfort

Institute of Inorganic and Analytical Chemistry

Goethe University, Max-von Laue-Str. 7

60438 Frankfurt am Main (Germany)
}

ed by reactive fragments (e.g. radicals) created in the organic film as a result of the primary ion impact.

Following these findings, ion-induced desorption experiments were performed for aromatic SAMs that form different structural phases on the $\mathrm{Au}(111)$ surface. ${ }^{[9]}$ These experiments unambiguously demonstrated that the ion-induced cleavage of chemical bonds in SAMs is extremely sensitive to details of their geometric and electronic configuration, due to the chemical reaction mechanisms involved. To further investigate this effect, a homologue series of 4,4'-biphenyl-substituted alkanethiols $\left[\mathrm{CH}_{3}-\mathrm{C}_{6} \mathrm{H}_{4}-\mathrm{C}_{6} \mathrm{H}_{4}-\left(\mathrm{CH}_{2}\right)_{n}-\mathrm{SH}\right.$; BPnS; $\left.n=1-6\right]$ deposited on a $\mathrm{Au}(111)$ substrate was analyzed. ${ }^{[10]}$ Former microscopic ${ }^{[11]}$ and spectroscopic ${ }^{[12,13]}$ experiments demonstrated that, depending on the parity of the number of $\mathrm{CH}_{2}$ units (the parameter $n$ is odd or even), two different structures of BPnS/Au(111) SAMs are observed, with higher packing density and more uprightly oriented molecules for the odd-numbered systems. Important$l y$, this odd-even structural effect coincides with the odd-even effects reported for the stability of these SAMs towards electrochemical desorption, ${ }^{[14,15]}$ exchange by other molecules, ${ }^{[16,17]}$ electron irradiation, ${ }^{[18]}$ and thermally induced phase transitions. ${ }^{[19-21]}$ In all these different aspects of the film stability, the odd-numbered systems were more stable than the even ones. Recent ion-induced desorption experiments for the BPnS/ $\mathrm{Au}(111)$ series demonstrated that the emission of the complete parent molecule, resulting from the $\mathrm{Au}-\mathrm{S}$ bond scission and the emission of the desulfurized parent molecule ([BPnS-S]), resulting from the $\mathrm{S}-\mathrm{C}$ bond scission, also exhibit clear oddeven effects. ${ }^{[10]}$ In this case, the odd BPnS/Au(111) SAMs show a systematically lower probability of the $\mathrm{Au}-\mathrm{S}$ bond scission with at the same time higher probability of the $\mathrm{S}-\mathrm{C}$ bond scission. Considering that the $\mathrm{Au}-\mathrm{S}$ bond has to be cleaved to allow for electrochemical desorption or for exchange by other molecules capable of SAM formation, previous electrochemi$\mathrm{cal}^{[14,15]}$ and exchange experiments ${ }^{[16,17]}$ indicated a higher chemical stability of this bond for odd systems. If this correlation holds, one could relate efficiency of ion-induced bond scission and chemical stability of the respective bond, as was suggested recently. ${ }^{[10]}$

However, it is known that the odd-even effect in BPnS/ $\mathrm{Au}(111)$ SAMs changes the packing density of the molecules and thus also the molecule-molecule interaction, which must significantly influence the electrochemical desorption and molecule exchange processes. Therefore, previous desorption experiments for BPnS/Au(111) SAMs alone could not unambiguously prove the direct relation between the efficiency of the ion-induced desorption process and the chemical stability of the corresponding chemical bond at the SAM-substrate interface. Such proof could be obtained by comparing desorption 
processes for SAMs in which the only difference in the energetics is related to the stability at the SAM-substrate interface. It would of course be desirable to perform such an analysis not only for a single analogue pair but for a whole family of molecules. Such an opportunity is provided by the homologue series of BPnSe/Au(111) $(n=2-6)$, which are selenolate analogs $(\mathrm{S} \rightarrow \mathrm{Se}$ substitution) of the BPnS/Au(111) SAMs (see Figure 1).

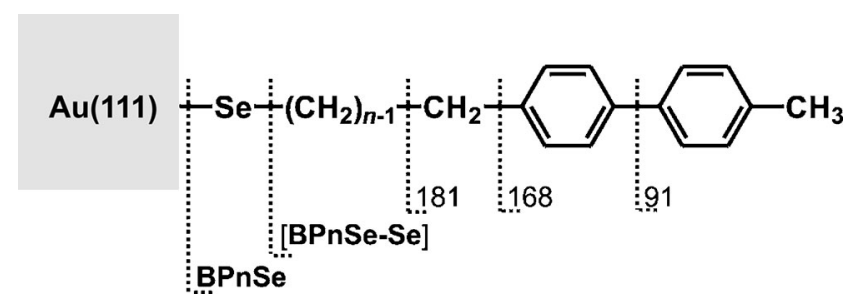

Figure 1. Schematic structure of the BPnSe/Au(111) SAMs with indicated chemical bonds that need to be cleaved for the emission of the parent molecule (BPnSe), the deselenized parent molecule ([BPnSe-Se]), and fragments corresponding to $m / z=91,168$ and 181 .

Previous microscopic ${ }^{[22]}$ and spectroscopic ${ }^{[23,24]}$ experiments confirmed that the packing density and structure of the $\mathrm{BPnSe} / \mathrm{Au}(111) \mathrm{SAMs}$ is essentially the same as for their thiol analogs. Following this similarity, recent exchange experiments comparing $\mathrm{BPnS} / \mathrm{Au}(111)$ and $\mathrm{BPnSe} / \mathrm{Au}(111)$ SAMs unambiguously demonstrated higher stability of the Au-Se bond as compared to the $\mathrm{Au}-\mathrm{S}$ bond in these SAMs. ${ }^{[17]}$

The mass spectra of photoionized neutral particles emitted with low kinetic energies ( $\left.10^{-2} \mathrm{meV}\right)$ following $15 \mathrm{keV} \mathrm{Ar}^{+}$ion beam bombardment of BPnSe/Au(111) SAMs $(n=2-6)$ are presented in Figures $2 \mathrm{~b}-\mathrm{f}$. Several molecular fragments that are common to all the spectra shown in Figure 2 are indicated in Figure 1 and correspond to the deselenized parent molecule
([BPnSe-Se]) and smaller fragments with $m / z=91,168,181$. For the present analysis, only two kinds of ions are of importance, namely the parent molecule (BPnSe) and the deselenized fragment of the parent molecule ([BPnSe-Se]). To compare the relative emission from different SAMs we have normalized the obtained mass abundances using the total detected amount of desorbed molecular material in the given spectra. The corresponding data are shown in Figure 3. To allow for direct comparison between $\mathrm{BPnSe} / \mathrm{Au}(111)$ and $\mathrm{BPnS} / \mathrm{Au}(111)$ SAMs, data obtained for [BPnSe-Se] and BPnSe emission (Figures $3 a, b)$ are confronted with the respective data obtained earlier ${ }^{[10]}$ for $[\mathrm{BPnS}-\mathrm{S}]$ and BPnS emission (Figures $3 \mathrm{~d}, \mathrm{e}$ ).

The most striking feature of the data obtained in the present experiment is the complete absence of parent molecule (BPnSe) emission in all spectra obtained for BPnSe/Au(111) SAMs. In contrast, parent molecule emission was observed in $\mathrm{BPnS} / \mathrm{Au}(111) \mathrm{SAMs}$ desorption, exhibiting a clear odd-even oscillation. ${ }^{[10]}$ This result shows that the probability of the ion induced $\mathrm{Au}-\mathrm{Se}$ bond scission must be radically lower compared to the $\mathrm{Au}-\mathrm{S}$ bond. Since recent exchange experiments ${ }^{[17]} \mathrm{dem}-$ onstrated that the stability of the Au-Se bond in these SAMs is higher compared to the $\mathrm{Au}-\mathrm{S}$ bond, we can now directly correlate the stability of the given chemical bond at the SAMsubstrate interface towards ion-induced desorption with the chemical stability of this bond.

The second feature clearly visible in Figure $3 a$ is the oddeven effect in the emission of the deselenized fragment [BPnSe-Se]. This effect is similar to what was observed earli$\mathrm{er}^{[10]}$ for [BPnS-S] emission (compare Figures $3 \mathrm{a}, \mathrm{d}$ ). This observation again is fully consistent with previous experiments revealing: 1) the same character of the odd-even structural effect in both types of homologue series, ${ }^{[22-24]}$ and 2) the same odd-even character in the stability for BPnSe/Au(111) and BPnS/Au(111) SAMs against the exchange by alkaneselenols
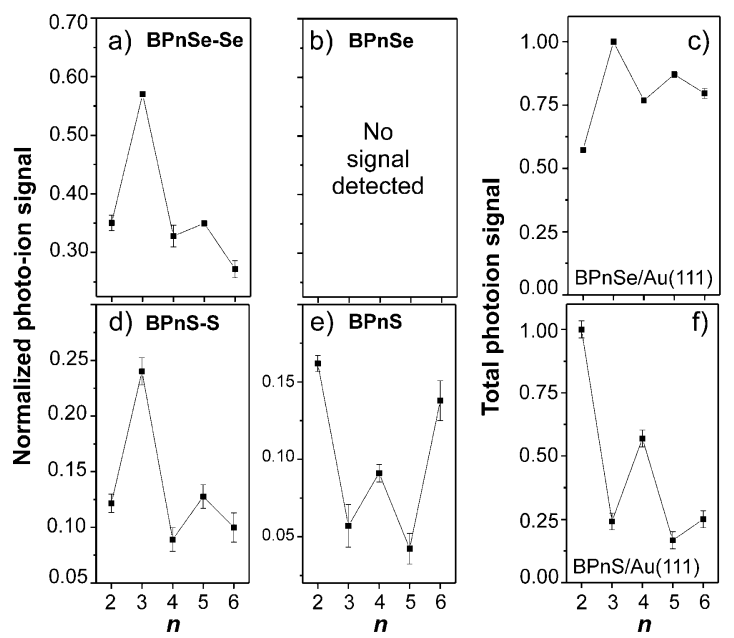

Figure 3. The normalized photoion signals for a) the BPnSe-Se fragments, b) the parent molecules (BPnSe), and c) the total detected signal plotted as function of the number of methylene units in BPnSe/Au(111) SAMs (a-c). To enable direct comparison, respective data reported by us previously (ref. [10]) for BPnS/Au(111) SAMs are presented in (d-f). Data in (a), (b), (d) and (e) are normalized to the total detected signal. Data in (c) and (f) are normalized to the maximal value.
Figure 2. Mass spectra obtained from photoionization of neutral molecular fragments with low kinetic energy desorbed during the $\mathrm{Ar}^{+}$irradiation of BPnSe/Au(111) SAMs. a) $n=2$, b) $n=3$, c) $n=4$, d) $n=5$, e) $n=6$.

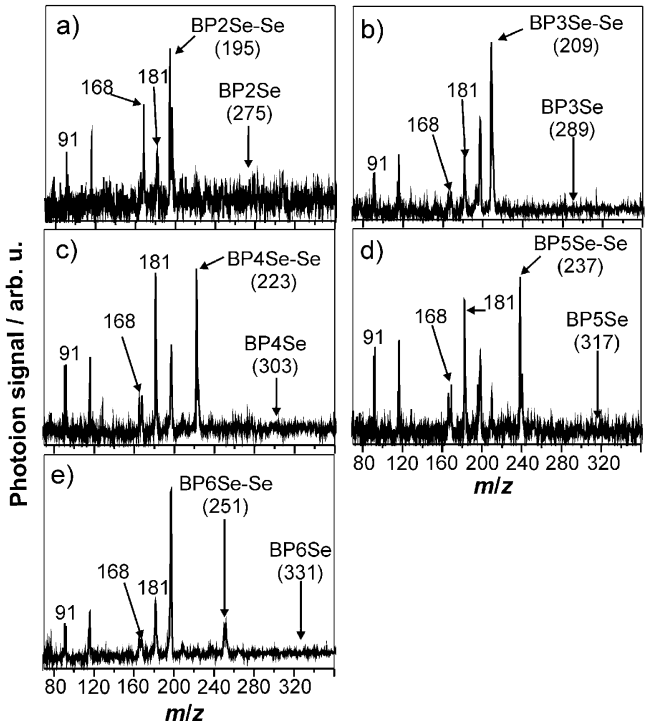

ChemPhysChem 2011, 12, 2554-2557

(c) 2011 Wiley-VCH Verlag GmbH \&Co. KGaA, Weinheim

www.chemphyschem.org

2555 
and alkanethiols, respectively (see Figure 5 in ref. [17]). Considering the present results, we can also directly conclude that the opposite sign of the odd-even effect for the [BPnS-S] emission as compared to BPnS (see Figures $3 d, e$ ) reflects that the increased chemical stability of the $\mathrm{Au}-\mathrm{S}$ bond results in decreased stability of the $\mathrm{S}-\mathrm{C}$ bond and vice versa. This is an expected chemical effect considering that the same $S$ atom is involved in both bonds which, apparently, can be traced by our desorption experiments.

The third feature is the odd-even effect in the total photoion signal detected for both homologue series (Figures $2 c, f$ ). In both cases the odd-even oscillation decays with increasing number $n$. This effect was also observed in corresponding exchange experiments and is fully consistent with the structural model of BPnS(Se)/Au(111) SAMs as discussed earlier. ${ }^{[17]}$ The opposite phase of this oscillation in desorption for BPnSe/ $\mathrm{Au}(111)$ and BPnS/Au(111) SAMs is directly related to the phase of the odd-even oscillation of the dominant contribution to the total photo-ion signal which comes from the deselenized parent molecule ([BPnSe-Se]) for $\mathrm{BPnSe} / \mathrm{Au}(111)$, and the parent molecule (BPnS) for BPnS/Au(111) SAMs.

Thermal desorption spectroscopy (TDS) has been used to probe the relative stability of the Au-S and Au-Se bonding in SAMs. The TDS experiments conducted by Witte et al. ${ }^{[25]}$ comparing benzenethiol (BS) and benzeneselenol (BSe) on $\mathrm{Au}(111)$ were interpreted by the authors as indicating higher stability of the $\mathrm{Au}-\mathrm{S}$ bond. However, this conclusion contradicts those of two other publications analyzing the stability of the same SAMs by competitive adsorption ${ }^{[26]}$ and electrochemical desorption. ${ }^{[27]}$ In our opinion the relative stability of the Au-S and $\mathrm{Au}-\mathrm{Se}$ bonds can be compared only for thiol and selenol SAMs analogs that form the same or very similar structures. Since the packing density of BS and BSe on $\mathrm{Au}(111)$ is different by about $35 \%,{ }^{[25]}$ such an analysis is not conclusive. More recently, however, Witte et al have conducted TDS experiments ${ }^{[28]}$ for anthracenethiol (AntS) and anthraceneselenol (AntSe) SAMs on $\mathrm{Au}(111)$ (see Figure S2 in the Supporting Information of ref. [28]), which have similar packing density and structure. ${ }^{[28,29]}$ Here the TDS data show no emission of the complete AntSe molecule from AntSe/Au(111) SAMs in the investigated temperature range $(350-700 \mathrm{~K})$ while deselenized fragment emission is observed with a peak at $478 \mathrm{~K} \cdot{ }^{[28]}$ For $\mathrm{AntS} / \mathrm{Au}(111)$, however, emission of the complete AntS molecule at $488 \mathrm{~K}$ (from the high-density phase) and the desulfurized fragment at $531 \mathrm{~K}$ (from the low-density flat-lying phase) was reported. ${ }^{[28,29]}$ These data (restricted to high-density phases for which such a comparison is possible) may indicate stronger $\mathrm{Au}-\mathrm{Se}$ bonding as compared to the $\mathrm{Au}-\mathrm{S}$ in these SAMs, which results in the emission of a deselenized fragment of the AntSe molecule (with Se atoms remaining on the surface as confirmed additionally by the XPS data ${ }^{[28]}$ for AntSe/ $\mathrm{Au}(111)$ and the emission of a complete AntS molecule for $\mathrm{AntS} / \mathrm{Au}(111) \mathrm{SAMs}$ in a similar temperature range. Such interpretation of TDS data obtained for AntS(Se)/Au(111) SAMs ${ }^{[28,29]}$ is consistent with desorption data shown herein, and in variance with the conclusions from the TDS data published in ref. [25].
In conclusion, our experiments unambiguously show that mass spectrometry of neutral fragments desorbed by ion bombardment of SAMs directly probes the chemical stability of the molecule-substrate interface in SAMs. Moreover, this new information together with the corresponding interpretation of the data obtained in a number of previous experiments, proves that the method proposed herein can qualitatively monitor changes in the stability of the molecule-substrate interface governed by three different aspects: 1) modification in the SAMs anchoring group ( $\mathrm{S} \rightarrow$ Se substitution, present data), 2) modifications in the chemical structure of the SAMs molecular backbone (the odd-even effects in BPnSe/Au(111) and $\mathrm{BPnS} / \mathrm{Au}(111)$ SAMs, present data and ref. [10]), and 3) modifications in the surface molecular packing for a given SAM (different structural phases in even BPnS/Au(111) SAMs, ref. [10]).

This qualitative information on molecule-substrate interface energetics should in turn enhance rational design and theoretical modeling of complicated and technologically relevant SAMs. For instance BPnS/Au(111) SAMs have been the subject of recent DFT calculations analyzing both bonding geometry ${ }^{[30]}$ and electronic structure ${ }^{[31]}$ at the molecule-metal interface, not revealing, however, any odd-even effect in the $\mathrm{Au}-\mathrm{S}$ bond stability visible in our desorption experiments. Also, relative stability of the Au-S and Au-Se bonding in SAMs as described herein was recently for the first time approached by DFT calculations, ${ }^{[32]}$ leading the authors to the general conclusion of a marginal difference in the stability. This is in contrast with desorption data presented herein, recent exchange experiments, ${ }^{[17]}$ and most of previous ${ }^{[26,27,33,34]}$ experimental findings. Therefore, we believe that the experimental results obtained for a well-characterized homologue series of $\mathrm{BPnSe} / \mathrm{Au}(111)$ and BPnS/Au(111) SAMs could be used as a reference for selecting an appropriate theoretical approach to model SAMsubstrate interface energetics.

Finally, we note that in the presented method, chemical reactions of reactive molecular fragments produced by the primary ion impact probe the chemical stability of the moleculesubstrate bonding in SAMs. Since a low dose of primary ions $\left(\sim 10^{10}\right.$ ions $/ \mathrm{cm}^{2}$, see the Experimental Section) is used for this analysis (static conditions), and the produced reactive species quickly move out of the primary ion impact zone, ${ }^{[8]}$ the proposed method analyzes, fast and locally, the unperturbed structure of the molecule-substrate interface in SAMs. This is in contrast to relatively slow thermal or electrochemical desorption experiments where, due to the global temperature ${ }^{[19-21,29,35]}$ or potential ${ }^{[36]}$ ramping, respectively, the molecule-substrate interface in SAMs can reconstruct before the desorption process takes place.

\section{Experimental Section}

$\mathrm{BPnSe} / \mathrm{Au}(111) \mathrm{SAMs}$ were formed by immersing gold-coated substrates in a $0.1 \mathrm{mM}$ ethanolic solution of the corresponding diselenide [BPnSe-SeBPn: $\mathrm{CH}_{3}\left(\mathrm{C}_{6} \mathrm{H}_{4}\right)_{2}\left(\mathrm{CH}_{2}\right)_{n} \mathrm{Se}-\mathrm{Se}\left(\mathrm{CH}_{2}\right)_{n}\left(\mathrm{C}_{6} \mathrm{H}_{4}\right)_{2} \mathrm{CH}_{3}$ with $n=2-6]$ for at least $24 \mathrm{~h}$. The synthesis of the molecules is described elsewhere. ${ }^{[37]}$ After incubation all samples were rinsed with ethanol and dried in a nitrogen flow. Atomically flat $\mathrm{Au}(111)$ sub- 
strates were epitaxially grown by thermal evaporation on aircleaved mica. ${ }^{[38]}$ The ion-irradiation experiments were performed in a UHV laser ionization mass spectrometer. Detailed information about the setup is available elsewhere. ${ }^{[39]}$ In brief, the experimental setup consists of an UHV chamber (base pressure below $2 \times$ $10^{-10} \mathrm{hPa}$ ), with an ion source and a time-of-flight mass spectrometer connected to it. Desorption was induced by a pulsed ( $500 \mathrm{~ns}$ ) $\mathrm{Ar}^{+}$ion beam ( $\left.15 \mathrm{keV}\right)$ directed onto the sample surface at an incidence angle of $45^{\circ}$. The experiments were performed in the static regime with a total ion fluence of about $8 \times 10^{10} \mathrm{ions} / \mathrm{cm}^{2}$. Laser post-ionization in combination with mass spectrometry was applied to ionize and detect neutral molecular fragments. The cloud of desorbed particles was intersected at a fixed distance of $4 \mathrm{~mm}$ parallel to the sample surface by a focused, pulsed $(6 \mathrm{~ns}, 10 \mathrm{~Hz})$ laser beam delivered by an optical parametric oscillator (QuantaRay MOPO-730) which was pumped by a Nd:YAG laser (Spectra Physics GCR-230). In order to reduce the photofragmentation process, resonance enhanced multi-photon ionization (REMPI) was used. To ionize the phenyl-containing molecular particles, resonant two-photon one-colour photoionization was used at $259 \mathrm{~nm}$ with relatively low photon fluence $\left(\sim 10^{18}\right.$ photons $\left./ \mathrm{cm}^{2}\right) .^{[40]}$ The created photoions were subsequently accelerated and detected in a linear time-of-flight mass spectrometer with a mass resolution of about 200. Changing the delay time between the incidence of the primary ion beam pulse on the sample and the firing moment of the laser, kept at a fixed position above the sample surface, enables selection of the kinetic energy of the analyzed species. To acquire mass spectra that result from ion-induced desorption by a chemical reactions mechanism, only low-energy molecular species where analyzed in the present experiments. Note that not all observed molecular fragments exactly correspond to a given $\mathrm{m} / \mathrm{z}$ value. Since small variations due to the mass calibration error can be safely excluded, we attribute the observed spread/deviation to the capture or loss of one or several protons during the desorption and/or the photoionization processes.

\section{Acknowledgements}

This work is financially supported by the Belgian Fund for Scientific Research, Flanders (FWO), the Flemish Concerted Action (GOA), Interuniversity Poles of Attraction (IAP) Research Programs, and the Polish Ministry of Science and Higher Education (0061/B/H03/2008/34). P.C. acknowledges support received from the Foundation for Polish Science under Homing fellowship.

Keywords: laser chemistry · mass spectrometry $\cdot$ monolayers · selenium $\cdot$ self-assembly

[1] J. C. Love, L. A. Estroff, J. K. Kriebel, R. G. Nuzzo, G. M. Whitesides, Chem. Rev. 2005, 105, 1103-1170.

[2] C. Vericat, M. E. Vela, G. Benitez, P. Carro, R. C. Salvarezza, Chem. Soc. Rev. 2010, 39, $1805-1834$.

[3] P. Maksymovych, O. Voznyy, D. B. Dougherty, D. C. Sorescu, J. T. Yates, Prog. Surf. Sci. 2010, 85, 206-240.

[4] R. L. McCreery, A. J. Bergren, Adv. Mater. 2009, 21, 4303-4322.

[5] D. E. Riederer, R. Chatterjee, S. W. Rosencrance, Z. Postawa, T. D. Dunbar, D. L. Allara, N. Winograd, J. Am. Chem. Soc. 1997, 119, 8089-8094.

[6] Z. Postawa, C. A. Meserole, P. Cyganik, J. Szymonska, N. Winograd, Nucl. Instrum. Methods Phys. Res. Sect. B 2001, 182, 148-154.
[7] E. Vandeweert, J. Bastiaansen, F. Verveacke, P. Lievens, R. E. Silverans, P. Cyganik, Z. Postawa, H. T. Rong, M. Buck, Appl. Phys. Lett. 2003, 82, 1114-1116.

[8] P. Cyganik, E. Vandeweert, Z. Postawa, J. Bastiaansen, F. Vervaecke, P. Lievens, R. E. Silverans, N. Winograd, J. Phys. Chem. B 2005, 109, $5085-$ 5094.

[9] F. Vervaecke, S. Wyczawska, P. Cyganik, Z. Postawa, M. Buck, R. E. Silverans, P. Lievens, E. Vandeweert, J. Phys. Chem. C 2008, 112, 2248-2251.

[10] F. Vervaecke, S. Wyczawska, P. Cyganik, J. Bastiaansen, Z. Postawa, R. E. Silverans, E. Vandeweert, P. Lievens, ChemPhysChem 2011, 12, 140-144.

[11] P. Cyganik, M. Buck, W. Azzam, C. Wöll, J. Phys. Chem. B 2004, 108, 4989-4969.

[12] K. Heister, H. T. Rong, M. Buck, M. Zharnikov, M. Grunze, L. S. O. Johansson, J. Phys. Chem. B 2001, 105, 6888-6894.

[13] H. T. Rong, S. Frey, Y. J. Yang, M. Zharnikov, M. Buck, M. Wühn, C. Wöll, G. Helmchen, Langmuir 2001, 17, 1582-1593.

[14] Y. T. Long, H. T. Rong, M. Buck, M. Grunze, J. Electroanal. Chem. 2002, $524,62-67$.

[15] I. Thom, M. Buck, Surf. Sci. 2005, 581, 33-46.

[16] T. Felgenhauer, H. T. Rong, M. Buck, J. Electroanal. Chem. 2003, 550, 309-319.

[17] K. Szelągowska-Kunstman, P. Cyganik, B. Schüpbach, A. Terfort, Phys. Chem. Chem. Phys. 2010, 12, 4400-4406.

[18] S. Frey, H. T. Rong, K. Heister, Y. J. Yang, M. Buck, M. Zharnikov, Langmuir 2002, 18, 3142-3150.

[19] P. Cyganik, M. Buck, J. Am. Chem. Soc. 2004, 126, 5960-5961.

[20] P. Cyganik, M. Buck, T. Strunskus, A. Shaporenko, J. D. T. E. Wilton-Ely, M. Zharnikov, C. Wöll, J. Am. Chem. Sci. 2006, 128, 13868-13878.

[21] P. Cyganik, M. Buck, T. Strunskus, A. Shaporenko, G. Witte, M. Zharnikov, C. Wöll, J. Phys. Chem. C 2007, 111, 16909-16919.

[22] P. Cyganik, K. Szelagowska-Kunstman, A. Terfort, M. Zharnikov, J. Phys. Chem. C 2008, 112, 15466-15473.

[23] A. Shaporenko, J. Müller, T. Weidner, A. Terfort, M. Zharnikov, J. Am. Chem. Soc. 2007, 129, 2232-2233.

[24] T. Weidner, A. Shaporenko, J. Müller, T. Schmid, P. Cyganik, A. Terfort, M. Zharnikov, J. Phys. Chem. C 2008, 112, 12495-12506.

[25] D. Käfer, A. Bashir, G. Witte, J. Phys. Chem. C 2007, 111, 10546-10551.

[26] F. K. Huang, R. C. Horton, D. C. Myles, R. L. Garrell, Langmuir 1998, 14, $4802-4808$

[27] Y. Sato, F. Mizutani, Phys. Chem. Chem. Phys. 2004, 6, 1328-1331.

[28] A. Bashir, D. Käfer, J. Müller, C. Wöll, A. Terfort, G. Witte, Ang. Chem. Int. Ed. 2008, 47, 5250-5252.

[29] D. Käfer, G. Witte, P. Cyganik, A. Terfort, C. Wöll, J. Am. Chem. Soc. 2006, $128,1723-1732$.

[30] G. Heimel, L. Romaner, J. Bredas, E. Zojer, Langmuir 2008, 24, 474-482.

[31] G. Heimel, L. Romaner, E, Zojer, J. Bredas, Accounts Chem. Res. 2008, 41, 721-729.

[32] E. de La Llave, D. A. Scherlis, Langmuir 2010, 26, 173-178.

[33] C. K. Yee, A. Ulman, J. D. Ruiz, A. Parikh, H. White, M. Rafailovich, Langmuir 2003, 19, 9450-9458.

[34] T. Weidner, A. Shaporenko, J. Müller, M. Höltig, A. Terfort, M. Zharnikov, J. Phys. Chem. C 2007, 111, 11627-11635.

[35] P. Cyganik, M. Buck, J. D. E. T. Wilton-Ely, C. Wöll, J. Phys. Chem. B 2005, 109, $10902-10908$.

[36] V. Mirsky, Trends in Analytical Chemistry 2002, 21, 439-450.

[37] J. Müller, A. Terfort, Inorg. Chim. Acta 2006, 359, 4821-4827.

[38] N. Vandamme, G. Verschoren, A. Depuydt, M. Cannaerts, W. Bouwen, P. Lievens, R. E. Silverans, C. Van Haesendonck, Appl. Phys. A 2001, 72, S177.

[39] E. Vandeweert, P. Lievens, V. Philipsen, J. Bastiaansen, R. E. Silverans, Phys. Rev. B 2001, 64, 195417.

[40] E. Vandeweert, C. A. Meserole, A. Sosteracz, Y. Dou, N. Winograd, Z. Postawa, Nucl. Instrum. Methods Phys. Res. Sect. B 2000, 164-165, 820826.

Received: July 8, 2011

Published online on August 25, 2011 